\title{
Bicarbonate supplementation as a preventive way in statins-induced muscle damage
}

\author{
Masaki Kobayashi, Toshiki Kagawa, Katsuya Narumi, Shirou Itagaki, Takeshi Hirano, Ken Iseki* \\ Laboratory of Clinical Pharmaceutics \& Therapeutics, Division of Pharmasciences, Faculty of Pharmaceutical Sciences, \\ Hokkaido University Kita-12-jo, Nishi-6-chome, Kita-ku, Sapporo, Japan
}

Received September 28, 2007, Revised October 17, 2007, Accepted November 12, 2007, Published January 9, 2008.

\begin{abstract}
Purpose. The aim of this study was to evaluate the bicarbonate-induced improvement of statins, cerivastatin, simvastatin acid and lovastatin acid -induced apoptosis using rat myoblast cell line (L6) as a model of in vitro skeletal muscle and of cerivastatin-induced muscle damage in vivo study. Methods. Statin-induced reduction of cell viability and apoptosis was measured by 3-(4,5-dimethylthiazol-2-yl)2,5-diphenyl tetrazolium bromide (MTT) assay and caspase assay. In vivo, we evaluated plasma creatine phosphokinase (CPK) level in cerivastatin-treated rat. Results. Bicarbonate prevented cerivastatin-, simvastatin- acid and lovastatin acid -induced reduction of cell viability, morphological change and caspase activation in L6 cells. Moreover, in the in vivo study, bicarbonate prevented cerivastatin-induced increase in CPK concentrations. Conclusions. These results from in vitro and in vivo studies support that bicarbonate supplementation prevented statin-induced muscle damage.
\end{abstract}

\section{INTRODUCTION}

HMG-CoA reductase inhibitors such as statins are the most widely used cholesterol-lowering agents for prevention of obstructive cardiovascular events [1-3]. However, one of the most important clinical adverse effects in therapy with statins is their skeletal muscle toxicity (rabdomyolysys) [4]. Skeletal muscle abnormalities can range from benign myalgia to myopathy, which is defined as a ten-hold elevation of the creatine kinase concentration [5]. When statins are prescribed as monotherapy, the incidence of myopathy is approximately $0.1-0.5 \%$ and are dose-related [6, 7]. The mechanism by which statins cause rhabdmyolysis is not precisely known though, the mechanism of statin-induced cytotoxicity has been investigated [8-10]. However, there have been few investigations on compounds suppressing statin-induced cytotoxicity. We previously found that bicarbonate suppressed cerivastatin-induced apoptosis in human prototypic embryonal rhabdomyosarcoma cell line (RD) as a model of in vitro human skeletal muscle [11]. However, it is unknown whether the bicarbonate preventing effect is a across-class observation so that other statins (e.g. simvastatin and lovastatin)-induced apoptosis and cerivastatin-induced muscle damage in vivo. In the present study, we evaluated the bicarbonate-induced improvement of other statins-induced apoptosis of L6 cell as a model of in vitro rat skeletal muscle and cerivastatin-induced muscle damage in vivo study.

\section{Materials and methods}

\section{Chemicals}

Cerivastatin $\mathrm{Na}$, simvastatin and lovastatin were kindly donated by Sankyo (Tokyo, Japan). Simvastatin acid and lovastatin acid were prepared from their lactone form by hydrolysis in a $0.05 \mathrm{~N} \mathrm{NaOH}$ solution, with stirring at $20^{\circ} \mathrm{C}$ for $30 \mathrm{~min}$. The hydrolyzed solution was adjusted to $\mathrm{pH} 7.4$ with $0.2 \mathrm{~N} \mathrm{HCl}$ and then stored $4{ }^{\circ} \mathrm{C}$ until use as described previously [12]. All other reagents were of the highest grade available and used without further purification.

\section{Animals}

Male Wistar rats, aged 7 to 8 weeks (200-350 g in weight), were obtained from Jla (Tokyo, Japan). The housing conditions were described previously [13].

Correspondence Author: Ken Iseki, Ph. D., Laboratory of Clinical Pharmaceutics \& Therapeutics, Division of Pharmasciences, Faculty of Pharmaceutical Sciences, Hokkaido University Kita-12-jo, Nishi-6-chome, Kita-ku, Sapporo, Japan. E-mail: ken-i@pharm.hokudai.ac.jp 
The experimental protocols were reviewed and approved by the Hokkaido University Animal Care Committee in accordance with the "Guide for the Care and Use of Laboratory Animals".

\section{Cell culture}

L6 rat skeletal myoblasts were maintained in plastic culture flasks (Corning Incorporated Corning) as described previously [14]. The L6 cells were kept in Dulbecco's modified Eagle's medium (Sigma) with 10\% fetal bovine serum (ICN Biomedicals, Inc., Aurora, $\mathrm{OH}$ ) and $1 \%$ penicillin-streptomycin at $37{ }^{\circ} \mathrm{C}$ under $5 \% \mathrm{CO}_{2}$.

\section{MTT assay}

3-(4,5-dimethylthiazol-2-yl) 2,5-diphenyl tetrazolium bromide (MTT) assay, a previously described method was used [15]. The MTT assay relies on the production of a colored formazan by the action of mitochondrial enzymes on MTT. For the MTT assay, L6 cells were seeded at density of $5-10 \times 10^{4}$ cells $/ \mathrm{mL}$ on 96 -well plastic plates. Following cell attachment (24 h), cerivastatin, simvastatin acid and lovastatin acid in combination with or without bicarbonate were added for the times indicated. At $4 \mathrm{~h}$ before the end of treatment, $10 \mu \mathrm{L}$ of PBS-containing MTT solution $(0.5 \%)$ was added, and the cells were incubated for a further $4 \mathrm{~h}$. The MTT medium was then replaced with $0.2 \mathrm{ml}$ dimetylsulfoxide, and absorbance was read at $590 \mathrm{~nm}$. Absorbance measured in MTT assays was expressed as percent of the control (defined as 100\%).

\section{Caspase assay}

Caspase assay was performed as described previously [11]. L6 cells were lysed with a cell culture lysis reagent (Promega, Madison, WI). Protein concentration of the cell lysate was adjusted to $5 \mu \mathrm{g} / \mathrm{mL}$, and the cell lysate was assayed for caspase-3/7 colorimetric protease assays measuring Ac-DEVD-pNa cleavage (Promega, Madison, WI) as described in the manufacturer's protocol (Promega, Madison, WI).

\section{Measurement of creatine phosphokinase (CPK) level}

The rats were anesthetized with sodium pentobarbital (40 mg/kg weight, i.p.). Cerivastatin solution with or without bicarbonate was injected through the femoral vein. Blood was collected at $0.5,1,2,3,4,6$ and $8 \mathrm{~h}$ after injection. Plasma was prepared by centrifugation (750 g for $10 \mathrm{~min}$ at $4^{\circ} \mathrm{C}$ ) of blood samples. The plasma samples were used to determine the CPK level, the skeletal muscle marker enzyme, by spectrophotometric measurement using a commercial kit (Wako Pure Chemical, Osaka, Japan).

\section{Data analysis}

Student's t-test was used to determine the significance of differences between two group means. Comparisons between more than two groups were made by using the post-hoc Scheffe test. Statistical significance was defined as $\mathrm{P}<$ 0.05 .

\section{RESULTS}

\section{Cytotoxicity of cerivastatin in L6 skeletal muscle cells}

Firstly, to clarify the mechanisms of the cytotoxicity of statins in vitro study, we examined the effects of cerivastatin on L6 cell viability. As shown in Fig. 1 (a), cerivastatin reduced the number of viable cells in time- and concentration-dependent manners. Based on these data, we calculated the $\mathrm{IC}_{50}$ values after exposure to cerivastatin for $48 \mathrm{~h}$ to be $2.39 \mu \mathrm{M}$. Next, we examined the morphological effects of cerivastatin on L6 cells. As shown in Fig. 1 (b), L6 cells underwent dramatic morphological changes in a concentration-dependent manner after exposure to cerivastatin for $48 \mathrm{~h}$. Then, we examined the activation of caspases. The effector caspase-3/7 plays a central role in apoptosis because it translocates from the cytosol into the nucleus upon activation [16]. As shown in Fig. 1 (c), cerivastatin markedly enhanced the activity of caspase-3/7 in a concentration-dependent manner. Moreover, we examined the effect of caspase- 9 inhibitor, zLEHD-fmk, on cerivastatin-induced caspase-3/7 activation. As shown in Fig. 1 (d), caspase-9 inhibitor blocked the activation of caspase-3/7. Accordingly, cerivastatin mainly induced apoptosis via a mitochondrial stress-induced cascade in L6 cells.

\section{Protective effects of bicarbonate on cerivastatin-induced apoptosis in L6 skeletal muscle cells}

We have previously reported that bicarbonate suppressed cerivastatin-induced apoptosis of RD cells [11]. Accordingly, we speculate that bicarbonate suppresses cerivastatin-induced apoptosis of L6 cells. 
(a)

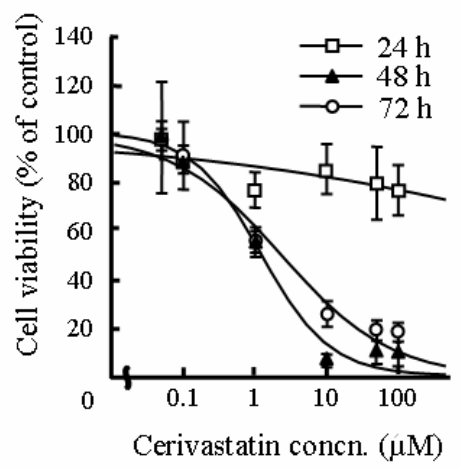

(c)

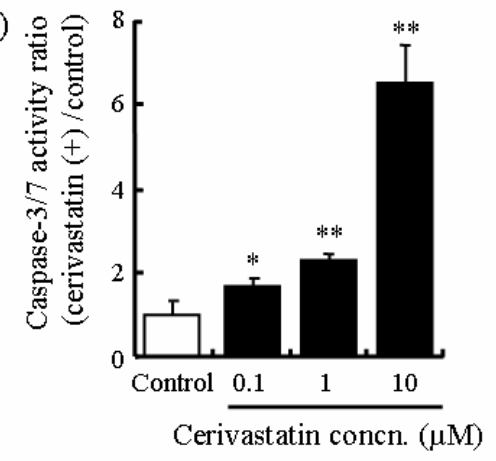

(b)
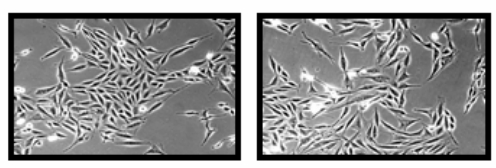

Control

Cerivastatin $0.1 \mu \mathrm{M}$
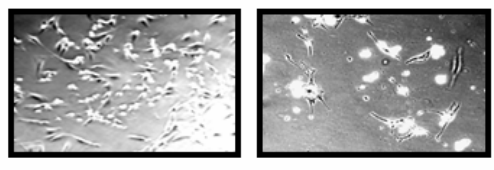

Cerivastatin $1 \mu \mathrm{M}$ Cerivastatin $10 \mu \mathrm{M}$

(d)

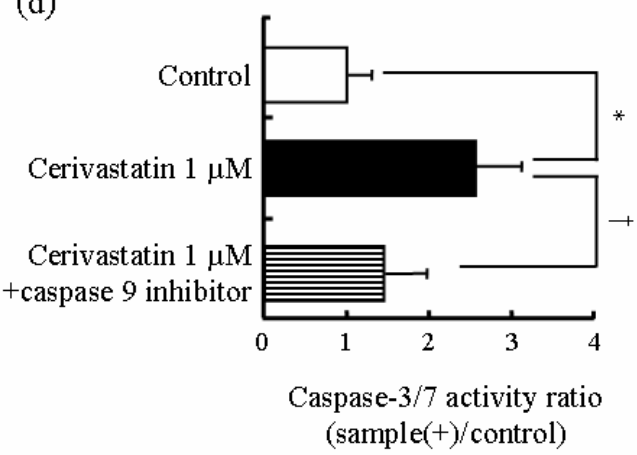

Figure 1. Effects of cerivastatin on the viability (a), morphology (b), caspase-3/7 activity ratio (c) of L6 cells and effect of caspase- 9 inhibitor on caspase-3/7 activity ratio in cerivastatin-treated L6 cells (d).

(a) Cell viability was measured by the MTT assay. Cells were exposed to various concentrations of cerivastatin for 24-72 h. Each point represents the mean \pm S.D. of 6 determinations. (b) Cells were exposed to various concentrations of cerivastatin for $48 \mathrm{~h}$, and the cells were examined by microscopy (x100). (c) Cells were exposed to various concentrations of cerivastatin for $48 \mathrm{~h}$, and the cell lysate was used to determine the caspase-3/7 activity ratio. Each column represents the mean with S.D. of 3-6 determinations. *; significantly different from control (no addition) at p < $0.05, * * ; \mathrm{p}<0.01$. (d) Cells were exposed to cerivastatin $(1 \mu \mathrm{M})$ in the absence or presence of $10 \mu \mathrm{M}$ caspase-9 inhibitor for 48 h. The conditions were identical to those described in the legend to Fig. 1 (c). Each column represents the mean with S.D. of 3-13 determinations. *; significantly different from control (no addition) at $p<0.01$, †; significantly different from cerivastatin alone at $\mathrm{p}<0.05$.

As shown in Fig. 2 (a), bicarbonate suppressed the reduction of cell viability induced by cerivastatin in a concentration-dependent manner within $25 \mathrm{mM}$. Accordingly, we subsequently used $25 \mathrm{mM}$ bicarbonate. The combination of cerivastatin and bicarbonate significantly prevented cerivastatin-induced caspase-3/7 activation, morphological change by cerivastatin alone (Figs. 2 (b,c)).
Protective effects of bicarbonate on other statins-induced cell injury in L6 skeletal muscle cells

We evaluated the bicarbonate-induced improvement of other statins, simvastatin acid and lovastatin acid-induced cytotoxicity. As shown in Fig. 3, simvastatin acid and lovastatin acid significantly reduced a number of viable cells and bicarbonate suppressed reduction of cell viability induced by simvastatin acid and lovastatin acid. Moreover, bicarbonate prevented simvastatin acid and lovastatin acid-induced caspase-3/7 activation. 
(a)

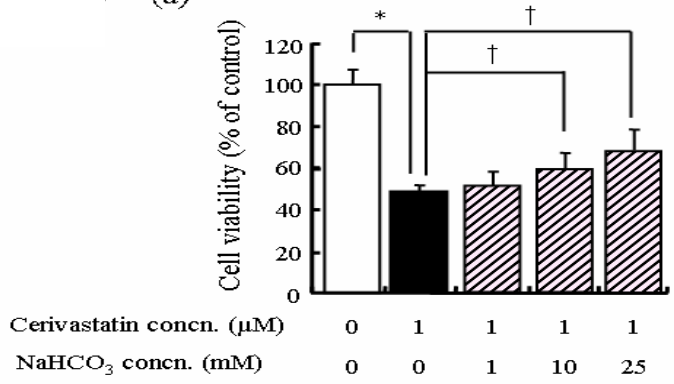

(b)

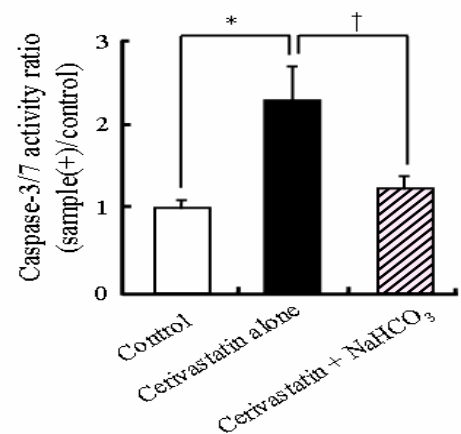

(c)

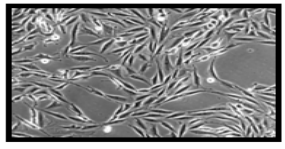

Control

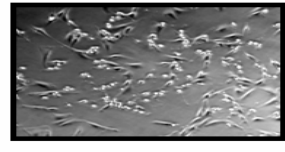

Cerivastatin alone

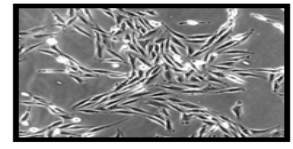

Cerivastatin $+\mathrm{NaHCO}_{3}$

Figure 2. Effect of sodium bicarbonate on reduction of cell viability (a), caspase-3/7 activity ratio (b) and morphological degeneration (c) in cerivastatin-treated L6 cells. (a) Cells were exposed to cerivastatin (1 $\mu \mathrm{M})$ in the absence or presence of various concentration of sodium bicarbonate $(\mathrm{pH} \mathrm{7.4)}$ ) for $48 \mathrm{~h}$. The conditions were identical to those described in the legend to Fig. 1 (a). Each column represents the mean with S.D. of 6 determinations. *; significantly different from control (no addition) at $\mathrm{p}<0.01$, $\uparrow$; significantly different from cerivastatin alone at $\mathrm{p}<$ 0.01 . (b) Cells were exposed to cerivastatin $(1 \mu \mathrm{M})$ in the absence or presence of $25 \mathrm{mM}$ sodium bicarbonate (pH 7.4) for $48 \mathrm{~h}$. The conditions were identical to those described in the legend to Fig. 1 (c). Each column represents the mean with S.D. of 3-6 determinations. *; significantly different from control (no addition) at $\mathrm{p}<0.01$, †; significantly different from cerivastatin alone at $\mathrm{p}<0.05$. (c) Cells were exposed to cerivastatin $(1 \mu \mathrm{M})$ in the absence or presence of $25 \mathrm{mM}$ sodium bicarbonate ( $\mathrm{pH} 7.4$ ) for $48 \mathrm{~h}$. The conditions were identical to those described in the legend to Fig. 1 (b).

We suggest that bicarbonate suppressed not only cerivastatin-induced apoptosis but also other statins-induced apoptosis.

\section{The improvement effect of bicarbonate on} cerivastatin-induced muscle damage in vivo. To evaluate bicarbonate-induced improvement of cerivastatin-induced muscle damage in vivo study, we tried to construct acute myopathy rat model. To avoid enterohepatic recirculation, we injected cerivastatin through femoral vein to construct acute myopathy model. Figure 4 (a) shows the plasma CPK levels after i.v. injection of $10 \mathrm{mg} / \mathrm{kg}$ cerivastatin. The CPK level was increased as time-dependently by $2 \mathrm{~h}$ compared with control. On the other hand, 0.1 and $1 \mathrm{mg} / \mathrm{kg}$ cerivastatin did not significantly affect plasma CPK level (data not shown). Next, to determine the effect of bicarbonate supplementation on the prevention of cerivastatin-induced myopathy, bicarbonate solution with cerivastatin was injected to rats. As shown in Fig. 4 (b), bicarbonate prevented cerivastatin-induced muscle damage in a concentration dependent manner.

\section{DISCUSSION}

To confirm the mechanism of cerivastatin-induced apoptosis in L6 cells, in vitro skeletal muscle model, we examined the effects of cerivastatin on viability, morphology and caspase-3/7 activation of L6 cells. Cerivastatin reduced the number of viable cells and caused dramatic morphological changes and caspase-3/7 activation in a concentration-dependent manner (Fig.1 (a-c)). Next, we focused on the molecular mechanism of inducing apoptosis by cerivastatin in L6 cells. It has been demonstrated that caspases are important mediators of apoptosis $(17,18)$. 

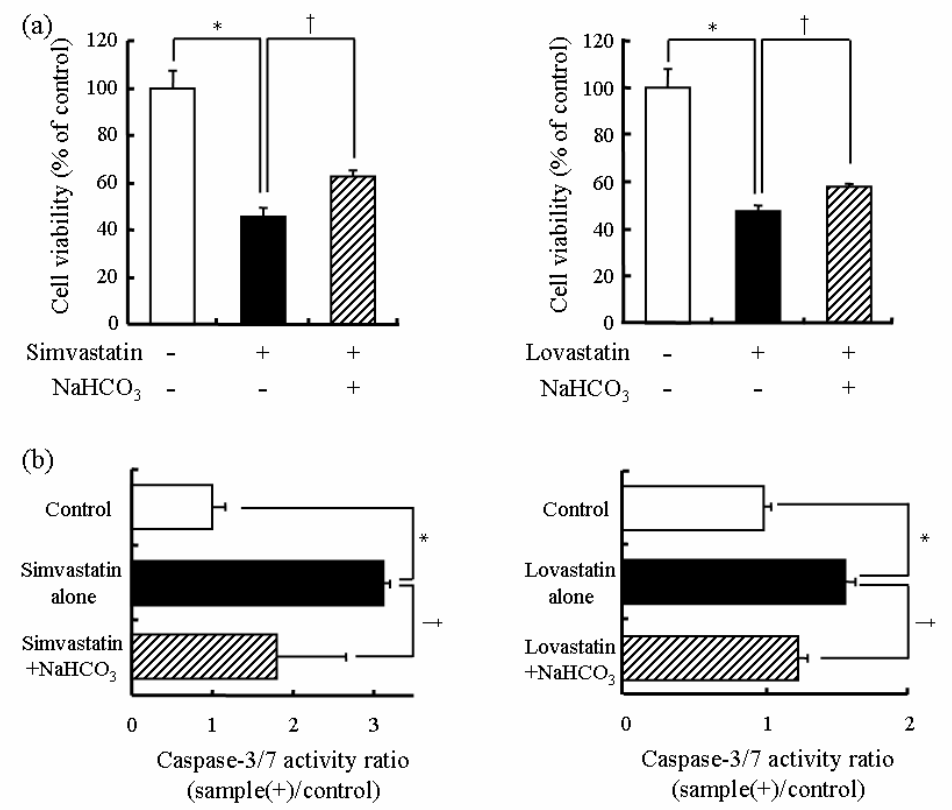

Figure 3. Effects of sodium bicarbonate on reduction of cell viability (a) and caspase-3/7 activity ratio (b) in other statins-treated L6 cells. (a) Cells were exposed to simvastatin acid (25 $\mu \mathrm{M})$ and lovastatin acid (50 $\mu \mathrm{M})$ in the absence or presence of $25 \mathrm{mM}$ sodium bicarbonate for $48 \mathrm{~h}$. The conditions were identical to those described in the legend to Fig. 1 (a). Each column represents the mean with S.D. of 3-6 determinations. *; significantly different from control (no addition) at $\mathrm{p}<0.01$. $\uparrow$; significantly different from simvastatin acid or lovastatin acid alone at $\mathrm{p}<0.01$.

(b) Cells were exposed to simvastatin acid $(25 \mu \mathrm{M})$ and lovastatin acid $(50 \mu \mathrm{M})$ in the absence or presence of $25 \mathrm{mM}$ sodium bicarbonate ( $\mathrm{pH} 7.4$ ) for $48 \mathrm{~h}$. The conditions were identical to those described in the legend to Fig. 1 (c). Each column represents the mean with S.D. of 3-7 determinations. *; significantly different from control (no addition) at p < 0.01 , $\uparrow$; significantly different from simvastatin acid or lovastatin acid alone at $\mathrm{p}<0.05$.

Various apoptotic stimulation activate the machinery; caspase-9 is activated by cytochrome c released from mitochondria. The activated caspase-9 converts inactive pro-caspase-3 into active caspase-3, resulting in chromosomal DNA fragmentation and cellular morphologic changes characteristic of apoptosis $[17,18]$. The activation of caspase-3/7 was blocked by caspase- 9 inhibitor zLEHD-fmk (Fig. 1 (d)). Accordingly, we suggest that the mechanism of cerivastatin-induced apoptosis is associated with mitochondrial stress-induced cascade in L6 cells.

To avoid severe adverse events, including myopathy and rhabdmyolysis, it is necessary to investigate the compound suppressing statin-induced apoptosis. In the present study, we therefore examined bicarbonate-induced prevention of statin-induced muscle damage from in vitro and in vivo study. Firstly, in vitro study, bicarbonate suppressed the reduction of cell viability induced by cerivastatin in a concentration-dependent manner within 25 mM (Fig. 2 (a)). Moreover, 25 mM of bicarbonate, prevented caspase-3/7 activation and morphological degeneration induced by cerivastatin (Figs. 2 (b, c)). Bicarbonate significantly suppressed other statins, simvastatin acid and lovastatin acid-induced L6 cell injury (Fig. 3 (a, b)). We have previously reported that l-lactic acid efflux is mediated by monocarboxylate transporter (MCT) 4 in RD and L6 skeletal muscle cells [14, 19]. 

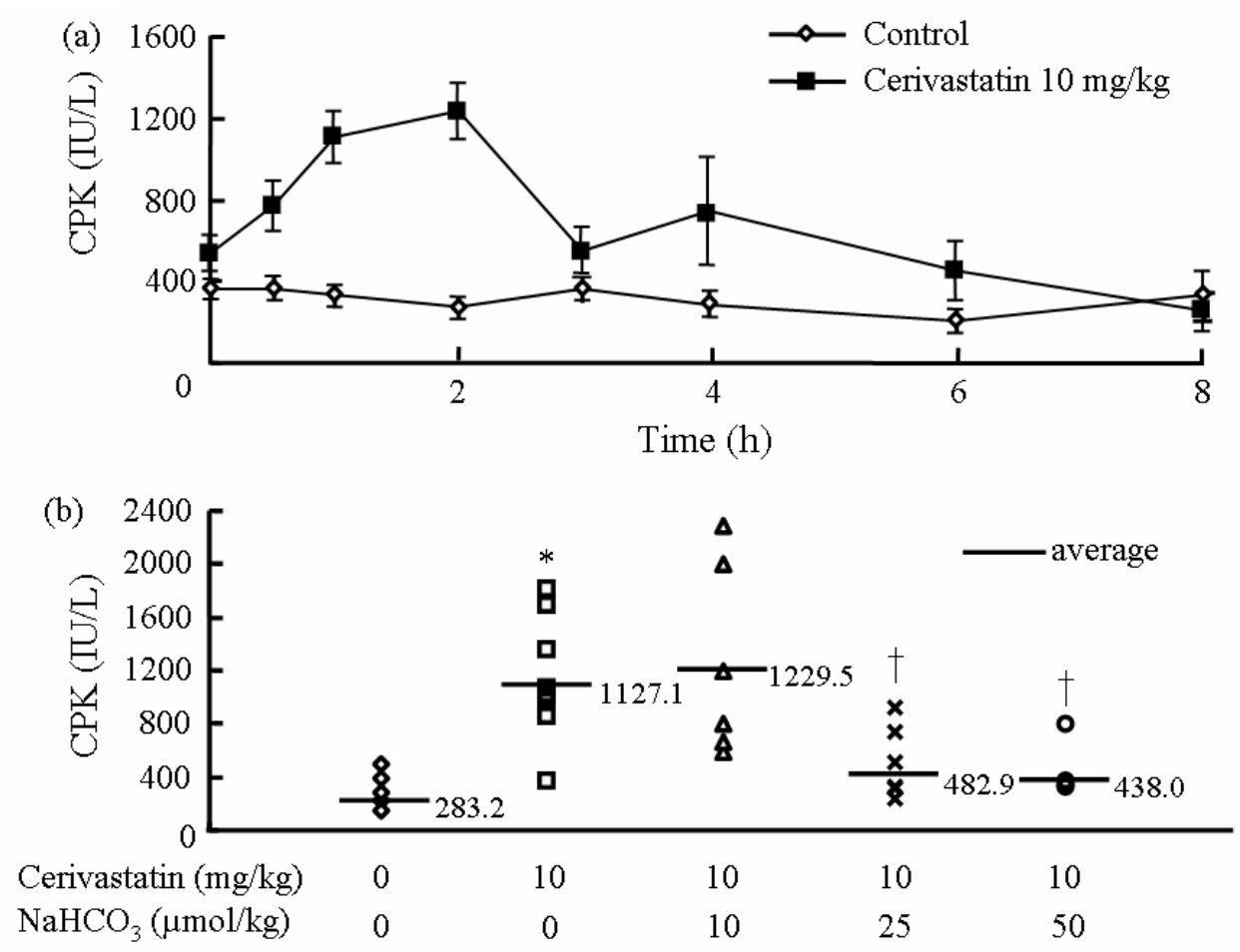

Figure 4. Plasma CPK levels after i.v. injection of cerivastatin (a) and effect of sodium bicarbonate on plasma CPK levels elevated by cerivastatin for $2 \mathrm{~h}$ after i.v. injection (b).

(a) CPK was measured as described in Materials and Methods. Each point represents the mean \pm S.E. of 4-7 determinations.

(b) The conditions were identical to those described in the legend to Fig. 4 (a). Each point represents the mean of 4-8 determinations. *; significantly different from control at $\mathrm{p}<0.01, \dagger$; significantly different from cerivastatin alone at $\mathrm{p}$ $<0.05$.

The efflux of l-lactic acid from skeletal muscle cells was significantly inhibited by lipophilic statins including cerivastatin, simvastatin acid and lovastatin acid. Moreover, we established a CD147 and MCT4 co-transfected cell line (cm) and examined the inhibitory effects of statins on l-lactic acid uptake by $\mathrm{cm}$. Lipophilic statins significantly inhibited l-lactic acid uptake concentration dependently [12]. Accordingly, inhibitory effects of statins on l-lactic acid transport mediated by MCT4 suggest the risk of leading to over-accumulation of the acid and disturbance of the muscle homeostasis, thereby, the possibility of inducing intracellular acidification and apoptosis in skeletal muscle.
We have previously reported that cerivastatin-induced apoptosis is associated with intracellular acidification and bicarbonate prevented cerivastatin-induced intracellular acidification [11]. We suggest that bicarbonate supplementation induces the intracellular alkalization, resulting from preventing statins-induced muscle damage.

Developing toxicological animal models for statin-induced rhabdomyolysis studies has been difficult. Pierno et al. [20, 21] have reported that pravastatin treatment at $100 \mathrm{mg} / \mathrm{kg} /$ day for 3 months does not produce any alteration of excitation-concentration coupling of the rat skeletal muscle. Moreover, Smith et al. [22] have reported that the incidence of induced myopathy 
in rats is dose-dependent, but the grade of degeneration was variable at a maximum dose of $2,400 \mathrm{mg} / \mathrm{kg} /$ day (on a bid regimen) of pravastatin for 2-4 weeks. The incidence was increased by concomitant intravenous administration of cyclosporin A at various doses for the same period, but the level of degeneration remained variable. We, therefore, tried to develop an acute myopathy rat model. Although cerivastatin was clinically administrated p.o, we injected cerivastatin through femoral vein in order to attain acute myopathy. The CPK level was increased as time-dependently by $2 \mathrm{~h}$ compared with control (Fig. 4(a)). On the other hand, 0.1 and $1 \mathrm{mg} / \mathrm{kg}$ cerivastatin did not affect plasma CPK level (data not shown). Next, to determine the effect of bicarbonate supplementation on the prevention of cerivastatin-induced myopathy, bicarbonate solution with cerivastatin was injected to rats. As shown in Fig. 4(b), bicarbonate prevented cerivastatin-induced muscle damage in a concentration dependent manner. In the present study, we have shown that the combination of statins and bicarbonate suppressed apoptosis of L6 cells by preventing reduction of cell viability and morphological change and decreasing caspase-3/7 activation. Moreover, bicarbonate prevented cerivastatin-induced muscle damage in our vivo study. However, the bicarbonate preventing effect by oral doses remains to be tested.

The strategy to combine statins with bicarbonate can lead to reduced risk of the severe adverse events including myopathy, rhabdmyolysis. Finally, this approach may prove to be inexpensive, safe, and useful to increase the efficacy of statins in hyperlipidemia patient therapy.

\section{REFERENCES:}

[1]. Havel, R.J., Rapaport, E., Management of primary hyperlipidemia. New Engl J Med,332:1491-1498, 1995.

[2]. Jukema, J.W., Bruschke, A.V., van Boven, A.J., Reiber, J.H., Bal, E.T., Zwinderman, A.H., Jansen, H., Boerma, G..J., van Rappard F.M., Lie, K.I. et al., Effects of lipid lowering by pravastatin on progression and regression of coronary artery disease in symptomatic men with normal to moderately elevated serum cholesterol levels. The Regression Growth Evaluation Statin Study (REGRESS). Circulation, 91:2528-2540, 1995.

[3]. Downs, J.R., Clearfield, M., Weis, S., Whitney,
E., Shapiro, D.R., Beere, P.A., Langendorfer, A., Stein, E.A., Kruyer, W., Gotto, A.M.Jr., Primary prevention of acute coronary events with lovastatin in men and women with average cholesterol levels: results of AFCAPS/TexCAPS. Air Force/Texas Coronary Atherosclerosis Prevention Study. JAMA, 279:1615-1622, 1998.

[4]. Omar, M.A., Wilson, J.P., Cox, T.S., Rhabdomyolysis and HMG-CoA reductase Inhibitors. Ann Pharmacother, 35:1096-1107, 2001.

[5]. Pedersen, T.R., Kjekshus, J., Berg, K., Haghfelt, T., Faergeman, O., Faergeman, G., Pyorala, K., Miettinen, T., Wilhelmsen, L., Olsson, A.G., Wedel, H., Scandinavian Simvastatin Survival Study Group. Randomised trial of cholesterol lowering in 4444 patients with coronary heart disease: the Scandinavian Simvastatin Survival Study (4S) 1994. Atheroscler Suppl, 5:81-87, 2004.

[6]. Garnett, W.R., Interactions with hydroxymethylglutaryl-coenzyme A reductase inhibitors. Am J Health Syst Pharm, 52: 1639-1645, 1995.

[7]. Maron, D.J., Fazio, S., Linton, M.F., Current perspectives on statins. Circulation, 101:207-213, 2000.

[8]. Guijarro, C., Blanco-Colio, L.M., Massy, Z.A., O'Donnell, M.P., Kasiske, B.L., Keane, W.F., Egido, J., Lipophilic statins induce apoptosis of human vascular smooth muscle cells. Kidney Int, 71:88-91, 1999.

[9]. Newton, C.J., Ran, G., Xie, Y.X., Bilko, D., Burgoyne, C.H., Adams, I., Abidia, A., McCollum, P.T., Atkin, S.L., Statin-induced apoptosis of vascular endothelial cells is blocked by dexamethasone. J Endocrinol, 174:7-16, 2002.

[10]. Ogata, Y., Takahashi, M., Takeuchi, K., Ueno, S., Mano, H., Ookawara, S., Kobayashi, E., Ikeda, U., Shimada, K., Fluvastatin induces apoptosis in rat neonatal cardiac myocytes: a possible mechanism of statin-attenuated cardiac hypertrophy. J Cardiovasc Pharmacol, 40:907-915, 2002.

[11]. Kobayashi, M., Kaido, F., Kagawa, T. Itagaki, S., Hirano, T., Iseki, K., Preventive effects of bicarbonate on cerivastatin-induced apoptosis. Int J Pharm, 341:181-188, 2007.

[12]. Kobayashi, M., Otsuka, Y., Itagaki, S., Hirano, T., Iseki, K., Inhibitory effects of statins on human monocarboxylate transporter 4. Int J Pharm, 317:19-25, 2006.

[13]. Itoh, T., Itagaki, S., Sasaki, K., Hirano, T., 
Takemoto, I., Iseki, K., Pharmacokinetic modulation of irinotecan metabolites by sulfobromophthalein. J Pharm Pharmacol, 56:809-812, 2004.

[14]. Kobayashi, M., Itagaki, S., Hirano, T., Iseki, K., Mechanism of L-lactic acid transport in L6 skeletal muscle cells. Drug Metab Pharmacokinet, 19:363-368, 2004.

[15]. Mosmann, T., Rapid colorimetric assay for cellular growth and survival: application to proliferation and cytotoxicity assays. J Immunol Methods, 65:55-63, 1983.

[16]. Ferri, K.F., Kroemer, G., Organelle-specific initiation of cell death pathways. Nat Cell Biol, 3:255-263, 2001

[17]. Salvesen, G.S., Dixit, V.M., Caspases: intracellular signaling by proteolysis. Cell, 91:443-446, 1997.

[18]. Thornberry, N.A., Lazebnik, Y., Caspases: enemies within. Science, 281:1312-1316, 1998.

[19]. Kobayashi, M., Fujita, I., Itagaki, S., Hirano, T. Iseki, K., Transport mechanism for L-lactic acid in human myocytes using human prototypic embryonal rhabdomyosarcoma cell line (RD cells). Biol Pharm Bull, 28:1197-1201, 2005.

[20]. Pierno, S., De Luca, A., Tricarico, D., Roselli, A., Natuzzi, F., Ferrannini, E., Laico, M., Camerino, D.C., Potential risk of myopathy by HMG-CoA reductase inhibitors: a comparison of pravastatin and simvastatin effects on membrane electrical properties of rat skeletal muscle fibers. J Pharmacol Exp Ther, 275:1490-1496, 1995.

[21]. Pierno, S., De Luca, A., Liantonio, A., Camerino, C., Conte Camerino, D., Effects of HMG-CoA reductase inhibitors on excitation-contraction coupling of rat skeletal muscle. Eur J Pharmacol, 364:43-48, 1999.

[22]. Smith, P.F., Eydelloth, R.S., Grossman, S.J., Stubbs, R.J., Schwartz, M.S., Germershausen, J.I., Vyas, K.P., Kari, P.H., MacDonald, J.S., HMG-CoA reductase inhibitor-induced myopathy in the rat: cyclosporine A interaction and mechanism studies, J Pharmacol Exp Ther, 257:1225-1235, 1991. 